\title{
Sustained Renewability: Approached by Systems Theory and Human Ecology
}

\author{
Tobias A. Knoch ${ }^{1,2}$ \\ ${ }^{1}$ Biophysical Genomics, Dept. Cell Biology E Genetics, Erasmus MC, Rotterdam, \\ ${ }^{2}$ BioQuant $\mathcal{E}$ German Cancer Research Centre (DKFZ), Heidelberg \\ 1 The Netherlands, \\ ${ }^{2}$ Germany
}

\section{Introduction}

With the growth of the world population and the ever-new technologies emerging from R\&D - both creating ever higher needs and expectations - also the energy amount to be acquired, stored, transformed, and finally used is exponentially growing and thus believed to be always at the limit. Actually this capability to use energy, has since the origin of our universe been the central drive of nature: first in its physical evolution, then in the evolution of biological life and finally in the emergence of human societies and cultures. In our modern industrialized life from primary food to industrial good production, via transport and information processing, to every form of cultural activity, everything is depending on this agent allowing the change of the physical state of matter or organisms. This is underlined by the fact that mass and energy are two sides of the same medal as shown by $\mathrm{E}=\mathrm{mc}^{2}$ (Einstein, 1905) and always conserved (Noether, 1918a, $1918 b)$. Without energy no work, no process, no change, and no time would exist and consequently the thirst for energy, surpasses the currently accessible resources by far. Interestingly, there is only one other basic resource, which might be equally important as matter and energy: information - the way of how energy is used for change. Also the information amount to be stored and processed is growing exponentially and believed to be always at the limit. Without doubt information technologies have become the key to success in nearly all sectors of modern live: R\&D is meanwhile mostly based on the storage and analysis of huge data amounts. In health care, diagnosis and treatment rely on imaging facilities, their sophisticated analysis and treatment planning. In logistics, the shipment of goods, water, electricity and fuels is driven by distribution management systems. The financial and insurance sectors are unthinkable without modelling. Finally, the IT sector itself is inevitably carried by the creation and manipulation of data streams. Thus, also here the demands outweigh the useable resources and especially the public sector struggles to increase their capabilities.

Limits showing e.g. syntropic/entropic materialistic, energetic or other barriers as those of the energy or IT sectors, are well known (Egger, 1975; Faber \& Manstetten, 2003). They have constrained first nature and later life since their beginnings and are one of the evolutionary drivers by the "survival of the fittest". Exponential demand growth until reaching a limit seems 
to be an inherent property of life and evolution in general (Faber, 1987). The other side of demand growth - waste and pollution - complies with this, although it is not using a resource but destroying the purity of another one. Obviously, this sustainability challenge beyond the materialistic regime can be found on all evolutionary levels up to the psychological, societal, and cultural level. All these levels act as a possible cause for exponential growth. Especially, the abilities of man in his modern societies have accelerated the use of common resources tremendously reaching the planetary carrying capacity (IPCC). Climate change and the sustainability challenge, thus is a complex combination of various effects, which in their holistic consequences have reached an unsustainable level threatening survival. The (Classic) Tragedy of the Commons (Hardin, 1968, 1994, 1998; Ostrom, 1990; Commons) describes this dilemma, in which (multiple) independently acting individuals due to their own self-interest can ultimately destroy a shared limited resource despite it is clear that it is not in the long-term interest of the local community or for the whole society. On universal time scales syntropy/entropy laws obviously predict that mankind will reach fundamental limits. Nevertheless, on short time scales huge resources are available: Already the sun delivers $\sim 3.910^{6}$ Exajoules to earth per year, i.e. $\sim 10,000$ times the current human energy consumption $\left(\sim 5.010^{2}\right.$ Exajoules/a). The natural geogene radioactive decay is also considerable and has kept the earth core molten now for 4.5 billion years. Both the energy inflow and outflow is balanced. Thus, with the little usage efficiency of our human societies of $\sim 10 \%$ the current renewable energy capacity surpasses the human consumption still $\sim 1$ million fold! Not only are those resources renewable on a human scale but also free of primary resource costs. Thus, more efficient usage of renewables here is undoubtedly the key to the further success of our societies.

Again there are striking similarities to the IT sector: Due to the pervasiveness of PCs, their number has grown beyond 1.5 billion, outweighing the capacity of computing centres $>100$ times. Since the capacity is peak performance oriented, less than $5 \%$ are used, i.e. $>95 \%$ of the capacity would be available $99 \%$ of the time. In a generic IT sense the term, a resource is any capability that may be shared and exploited by a network - normally termed "grid". These resources have been already paid for including their external follow-up costs (environmental etc.). The same holds to less extent for cluster infrastructures due to virtualization strategies. The Erasmus Computing Grid (de Zeeuw et al., 2007) with $\sim 20,000$ PCs ( 50,000 cores, $\sim 50$ Teraflops), corresponds to a $\sim 30 \mathrm{M} €$ investment. Especially in the notoriously under-funded public domain more efficient resource usage by means of grid would satisfy a big demand challenge. Thus, both in the energy, IT, as in any resource sector more efficient usage is of major importance for advancements. Thus, at least locally the disaster of reaching the (physical) limit can be delayed largely. A prime example from the production of fundamental raw materials is e.g. the integrated production in the chemical industry (Faber et al., 1987): Here byproduct usage, i.e. the waste of one process, is reused in another one as basic resource or often even as main process component (Jentzsch, 1995). Integrated production can reach the level of an extremely fine-tuned ecological organism (as in the highly sophisticated chlorine chemistry) that little changes have severe "survival" consequences for the whole system (Egger \& Rudolph, 1992; Faber \& Schiller, 2006). In real biological systems, however, there is more flexibility as in the highly integrated and sophisticated agro-forestry systems e.g. in Indonesia, which have been developed over centuries reaching extremely high efficiencies and are one of the biggest cultural achievements ever. In both cases the efficiency, i.e. the relation between system input and output, are maximized and beat every other process or management (Faber et al., 1998). 
Here, the internalization challenge of underused energy resources in general and especially of the vastly underused renewable energies is analysed by the new concept of Sustained Renewability combining systems theory with Human Ecology and describing adequately the integrated holistic ecology like system parameters and strategies necessary. Therefore, fossil, renewable energy as well as grid and cloud IT resources (Foster \& Kesselmann, 2004), their exploitation networks and organizational exploitation structures are analysed generically in relation to their technical systemic challenge. To approach the internalization challenge of underused renewable resources, the novel generic notion of the Inverse Tragedy of the Commons, i.e. that resources are underused in contrast to their overexploitation, is introduced. It is combined with the challenges on the micro level of the individual with its security/risk/profit psychology (Egger, 2008) as well as on the macro level of autopoietic social subsystems (Egger, 1996; Luhmann, 2004, 2008; Maturana \& Varela, 1992). To derive points of action, the classical Human Ecology framework (Bruckmeier \& Serbser, 2008; Egger, 1996) will be extended to describe the interactions between invironment-individual-society-environment completely and then is combined with the systemic complexity challenge. This leads inevitably to the new concept of Sustained Renewability and defined point of actions. Thus, sustained systemic renewability of resources in general can be really reached and thus leaves at least on the human scale much room for advancement for a big part of our future.

\section{Fossil and renewable energy resources and their means of exploitation}

Energy is always bound to and thus stored in a state of matter and has to be extracted thereof and transformed into the corresponding form for a certain usage. Primarily the energy we have access to comes either from nuclear fusion as in our sun (heating and driving the atmosphere), from nuclear decay within earth (keeping a molten core, volcanism, plate tectonics), and from the gravitational fields of our planetary system (tidal changes). This primary access is far from endless or renewable: e.g. hydrogen fusion has been done $2 / 3$ already, i.e. only $\sim 2$ billion years are left for hydrogen fusion and thus already in $\sim 300$ million years the earth atmosphere will start to be heated up so much that life as one knows cannot exist anymore. Radioactive decay and the gravitational energy are also slowly used up. Consequently, the term renewable in that sense is only a relative terminology in respect to human time scales: Considering sun energy present for another 100 million years means $\sim 30$ million human generations or $\sim 30$ times the evolutionary development to homo sapiens. Nevertheless, on a human scale the term renewable thus really makes sense. In contrast, fossil energy resources (despite geogene gas and radioactives) consist mainly of organic substances produced through biogene conversion of sun energy by photosynthesis and their further transformation by geological process to coal, gas and oil. I.e. they are in principle a tertiary energy resource already. Due to the slow geogene processes and geological exploitation degree, the accessible size of these resources is fairly limited and especially concerning the human energy consumption very limited compared to the size of primary energy resources, their lasting and also not changeable natural production. Also the forms of energy which are termed renewables are in that sense secondary resources: i) sun energy is stored in photons, i.e. light, ii) wind energy is due to the sun energy transformed to heat creating atmospheric pressure imbalances, iii) hydro energy is due to water evaporation and gravitational lifting to higher altitudes and rain, iv) tidal energy is based on the earth-moon gravitational energy and stored in ocean movement, 
v) geothermal energy is heat from radioactive decay stored in the geosphere itself, and vi) biomass is sun energy transformed by photosynthesis into biological matter as e.g. wood.

\subsection{Renewable energy resources and their distributed exploitation}

Renewable energy resources are due to their primary and secondary origins in principle homogenously distributed in an extensive and variant mixture compared to the very localized fossil resources: i) sun energy depends mostly on the geographic altitude, ii) wind energy is strong at coasts, great plains or mountains, iii) hydro electric energy needs rain, mountains, or rivers, iv) tidal energy needs tidal differences, v) geothermal energy is best at geological active sites, and vi) biomass counts on a vivid agro- and forestry capability, i.e. thus fertile soils and water. Actually in biological terms the presence of ample energy resources at each location, which are available in principle everywhere and in principle exploitable similarly, are the deeper basis for the thriving of life, i.e. the success of evolution (neglecting now extreme life forms). Three different exploitation means of these renewables can be distinguished e.g. in electricity production: i) direct conversion of e.g. sun energy by photovoltaics or heat by thermotaics, ii) conversion of kinetic energy via a generator as for hydro, tidal, or wind power, and iii) chemical conversion into heat as for biomass or directly for geothermal power, then into kinetic energy before electricity generation. Consequently, to reach the highest exploitation and conversion efficiency it is obvious to use the local resource mixture according to the usage profile, and only transport the overproduction to where it currently might be needed or stored for local demand rich times, i.e. to secure supply for the peak demand. Thus, here also the most systemic integration, that means the best adaption to the local usage scenarios can be reached, since the conversion plants, i.e. photovoltaic modules, wind turbines, small hydroelectric plants etc. are relatively small in size and can be aggregated in the most modular and thus sensible way. Also the exploiters and/or producers are either the same as the users or at least very near to them, thus ownership and participation in the exploitation-transformation-usage cycle can be maximal.

\subsection{Fossil energy resources and their central exploitation}

In contrast, the fossil resources are highly localized due to their origin: coal, gas, oil and uranium deposits are regional and need with their decline increased exploitation efforts. Despite being a local resource in a globalized world they are transported to the power plants. Thus, local economic thriving depends on an efficient transport system. Fossil energy conversion is based on chemical (in the case of nuclear decay, physical) transformation into heat, which is then transformed into kinetic energy driving turbines connected to generators for electric energy. Whereas renewable conversion is high-tech, the latter is still based on the steam engine and the electric generator. The best efficiencies are reached for big plants or a systemic combination of electricity and heat. Thus, due to the size the transformed energy transport itself becomes a major challenge and cost factor due to the large losses involved. From evolutionary optimized biological systems it is known that their scaling and success is based on: i) the distribution system is fractal, ii) the transport loss is minimized, and iii) the smallest part of the transport system has the same minimum size. Unfortunately, for the modern distribution networks this is mostly due to redundancy issues not the case anymore. Due to the plant size and the transport issues, the investments are high and only doable by international private companies, with relatively low integration with the local usage structures or participation of the local users. Thus, the production and usage can hardly be 
integrated in a systemic manner anymore with high efficiency. Beyond, fossil resources have one big drawback: they produce waste, i.e. $\mathrm{CO}_{2}$ is the leftover, whereas renewables only convert the energy form but not a resource additionally to the energy form. Thus, in a limited world this unavoidable leads to pollution and thus e.g. climate challenge.

\section{Generic organization of the fossil and renewable energy sectors}

As described briefly before, there are huge renewable energy resources available, which are based on the earth own geological nuclear decay, the suns nuclear fusion energy reaching us as light, and planetary gravitation. Simultaneously, there is a great shortage of exploitable resources as constantly claimed by users and providers - similar to the IT sector. Consequently, this paradoxical situation must have a reason, despite even the relative slow turnover rates of technical solutions in the energy sector, which are $\sim 30-50$ years for a production facility and perhaps the double for a complete new technology generation, compared to the 3-5 year fast turnover rates for a full technology replacement cycle in the IT sector. Thus, comparing the production solutions and organization of fossil and renewable energy resources is important. Both are based on dedicated organizations which handle the technical as well as management challenges and posses the same fundamental organization principles similar to the IT sector: i) ownership and control, ii) size of plant, iii) diversity and distribution, iv) technological broadness, and v) spatial distribution. To understand further the challenges, which still exist despite the crucial longing for energy and IT, the main three different electricity production approaches in Germany are analysed:

The renewable energy sector has grown tremendously in Germany in the last 10-15 years mainly by guarantying a fixed price for the produced energy allowing return of investment of $\sim 6 \%$ per year over 20 years: Today $\sim 25,000$ wind turbines with $\sim 30 \mathrm{GW}$ peak performance and $\sim 800,000$ photovoltaic plants with $\sim 18$ GW peak performance of electricity deliver $\sim 7 \%$ and $\sim 3.5 \%$ of the German electricity consumption. Together with biogas and biofuel production, combined heat and electricity production (KWK) and hydroelectric plants from lakes and rivers - each from some $\mathrm{kW}$ to some MW peak capacity - in total $\sim 17 \%$ of the German electricity are now renewable and emission free. Whereas wind mills have a peak performance of $300 \mathrm{~kW}$ to 7.5 MW and are usually aggregated in parks of up to 50-100 mills, photovoltaic plants range from 1-2 kW to $\sim 30-50 \mathrm{MW}$ peak performance. Wind parks naturally reside in wind rich regions but are meanwhile spreading to the southern continental regions. Photovoltaic plants are installed throughout the country on the roofs of private households, government, or industry buildings. Bigger ones are also placed on farmland and conversion zones e.g. unused industrial estates. Investment costs range from some thousand Euros for a photovoltaic plant on a family home, some million for a medium sized windmill, to some hundred million for a big photovoltaic plant or wind park. Consequently, the production plants fit different business models and investor groups from the individual up to institutionalized funds. The electricity is mainly introduced into the grid and has priority by law over conventional electricity production. The electricity grid providers measure the production and the producers are monthly refunded by the local grid or electricity company. The grid belonged to the four big German electricity companies ENBW, EON, RWE, and Vattenfall until recently, but is now in other private hands. The free energy flow to the consumer - so called grid-neutrality - is guarantied by law. The price guaranty to the producer is shared by an addition to the bill of all electricity consumers in a social manner and often also sold as special green electricity product then by green energy sellers. Besides the knowledge gain in Germany and being the world 
leader in renewable energy facility production with $\sim 350,000$ employed people meanwhile, the resource, i.e. sun or wind, has not to be paid for, which leads to a big economic advantage. Due to the range of business models in principle everybody can be an electricity producer, which means a democratization of electricity or renewable energy production within society.

The public city producers, which often have been owned by the cities or regions especially in the past have a very conventional portfolio consisting of coal or gas power plants, which are sized to serve the local or regional electricity and sometimes thermal, i.e. heating, energy needs. Historically they developed when electricity and heat was starting to be needed by major parts of society, i.e. between 1850 and 1950. The electricity is put into the electricity grid, which has often belonged also to the public city producers. The distribution network for the heat, which is a byproduct of the conventional electricity production, has also been build up by them, since this was relatively easy to implement concerning the technical and organizational efforts for a well thermally isolated pipeline system underground from production to consumers throughout a city. Electricity, nevertheless, is mainly traded at the European Energy Exchange and production depends on the national demand price, which depends again on the coal, oil, and gas trading prices, i.e. depends on a European/worldwide market price and thus is a major part of the production costs. The local city producers are also the major seller for their electricity. Meanwhile, many of them possess also renewable energy production capabilities (photovoltaic plants or wind parks, usually regional), besides the classic hydroelectric production facilities at lakes and rivers, which again has regulatory reasons. Since they are connected to the regional government and thus are controlled by the local inhabitants they are relatively much bound into the regional development process as well and also impact the regional industry.

In contrast, the four large-scale producers of electricity in Germany - ENBW, EON, RWE, and Vattenfall, who are often termed the big "German Four" - are meanwhile world wide acting producers of mainly conventional coal, gas, oil, and also atomic electric power. Their plants have investment costs of billions and their regional placement depends besides the energy production process and consumption needs mostly on business and regulatory reasons. Thermal energy is only in some cases used locally for heating since the amount surpasses by far the local demand, thus the electricity, which is put to the electricity grid, is often internationally transported through the network to the consumer. The network for a long time mainly belonged also to them until recently, and had been bought from regional city producers over the years, thus the "German Four" controlled production and transport in a very monopolistic manner. Naturally, they also have to buy the energy resource and thus depend critically on the resource price of energy resources, although due to their size they are in the position to influence that by their large demand. In selling terms they are the big sellers of electricity and due to their market position (and especially while being owners of the distribution network) can influence the price to some extent to their gusto. That this is not excessively abused, the German government has implemented a regulation agency controlling their market and price models. Due to the unavoidable switch to renewables due to the climate challenge they also invest meanwhile into very large photovoltaic plants, wind parks especially offshore, and hydroelectrics - again based on their business model of large-scale with a monotechnic approach. According to their financial power they act such that their market position, i.e. their monopolistic centrality is hardly touchable and thus that they can control the heart of the electricity sector in Germany. The dependencies this creates and the risk for society is retrospectively also one reason for the huge success of renewables with their decentral relative small-scale and thus democratic production. 


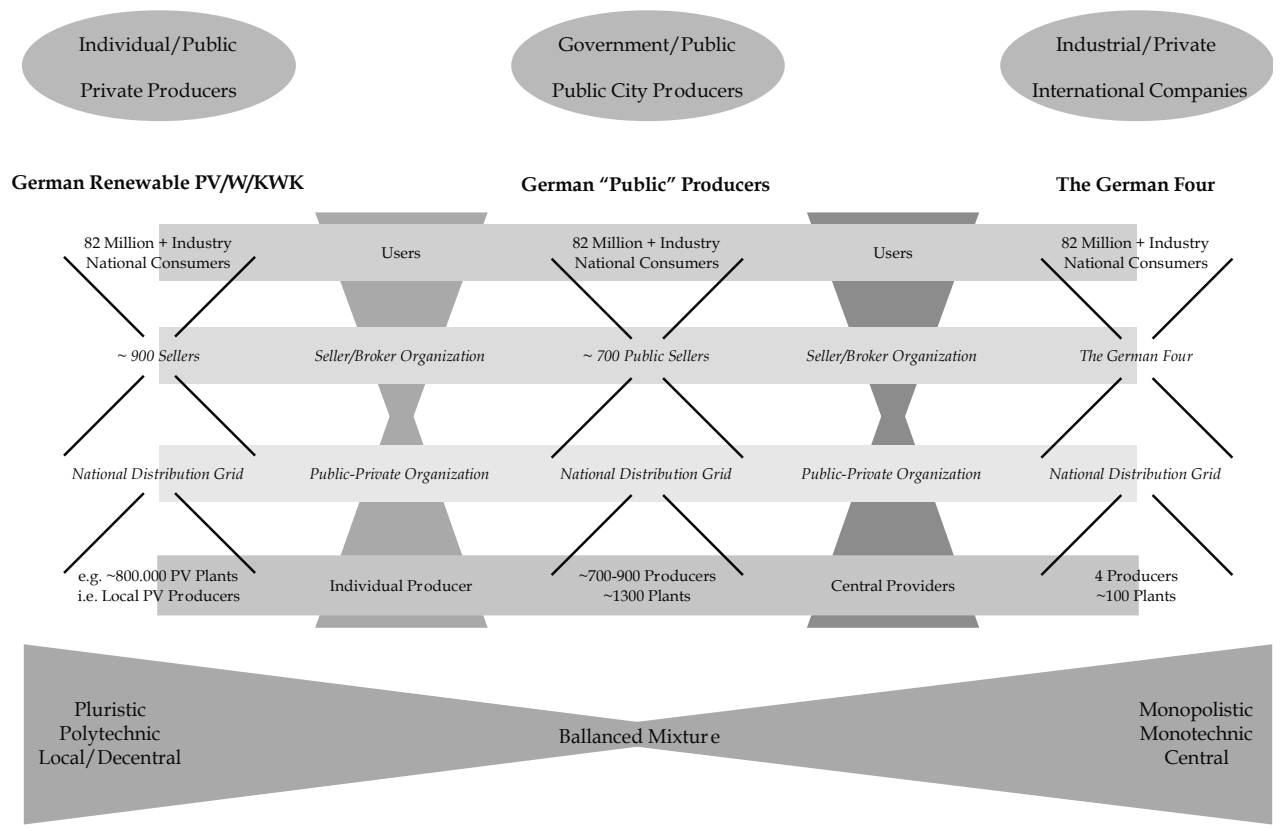

Fig. 1. Abstraction and detailed structure of the German electricity sector, showing three pillars and the four levels of infrastructures involved from production to usage. The three pillars are characterized by: i) individual/public "private" producers, ii)

government/public city/regional producers, and the very few industrial privately owned international companies. All share four levels of infrastructure from production to usage: i) users, ii) seller organizations, iii) the semi-private, i.e. public-private network organizations, and iv) individual producers. The three pillars are already characterized by their means of energy production: i) renewable and small scale, ii) regional and medium sized, and iii) classic large scale fossil and atomic. Whereas the first can be characterized by pluristic, polytechnic local/decentral means, the last is characterized by monopolistic, monotechnic, and central terms. Although, the details may vary, the structure leads to similar challenges on the micro and macro level, which can be understood by the Human Ecology rectangle

Generalizing, the renewables obviously belong to the class of individual/public distributed producers with a pluristic, polytechnic and local/decentral approach, whereas the "German Four" large scale producers are clearly industrial/private with a monopolistic, monotechnic, and central attitude. The German government/public city producers are a mixture of both: government and public, not too pluristic, polytechnic, and local/decentral and neither industrial/private, nor monopolistic, monotechnic, and central. Consequently, this shows already the similar property and power structures in the energy and IT sectors. Especially the "German Four" show the similarity to the newest development concerning IT resources, i.e. clouds, with the same monopolistic structures etc. and blocking effects on development. The analysis of these and other such many an infrastructure shows that four levels of organization are involved also in energy producing and distributing organizations: i) users, 
ii) organizing broker organizations, iii) provider organizations, and iv) individual providers. In a more abstract form this shows that actually there are i) individuals and ii) societies of individuals, which are both involved on each of the four levels of organization, with a different degree of influence. Consequently, there is a micro level from which a macro level emerges, having again an influence on the micro level, i.e. that both levels are connected in a complex and cyclical manner as in any evolutionary evolving system. Thus, the micro level is constituted by an invironment and the macro level creates an environment. This will later constitute already the Human Ecology rectangle.

\section{Generic organization of grid and cloud IT infrastructures}

Obviously, there are also huge resources available in the IT sector - similar as in the renewable energy sector, although there is - at the same time - a shortage of resources as constantly claimed by users and providers. Consequently, this paradoxical situation must have again a reason and especially for the IT sector where the opportunities for technical solutions with fast turnover rates of 3-5 years for full technology replacement cycles are large compared to the $\sim 30-50$ years in the energy sector. Grid and cloud infrastructures are one solution to ease the resource shortage by more efficient usage of available resources and are based on dedicated organizations, which handle the technical as well as management challenges involved. They also posses the same fundamental organization principles and can be classified by the same characterization as already the energy sector: i) ownership and control, ii) size of grid/cloud, iii) diversity and distribution, iv) technological broadness, and v) spatial distribution. Thus, it is very interesting to see that despite the much higher turnover rates and the innovative potential of the IT sector in principle the same challenges exist as in the energy sector. Therefore, now two grid and one cloud infrastructure will be investigated in greater detail to show the similarities:

The Erasmus Computing Grid (ECG) is one of the largest desktop grids for the biomedical research and care sectors worldwide (de Zeeuw et al., 2007; Fig. 2). The computing cycles of the desktop computers of the Erasmus Medical Centre and the Hogeschool Rotterdam (the local University for Applied Sciences) are donated to the ECG. Technically, these cycles are exploited by the middleware CONDOR and a newly developed management system, which administrates on the one side all the computers in the grid as well as the users and on the other hand posses an easy accessible back-end/front-end system for usage. The latter is especially important for efficient use and security: The users only need to deliver their application, which then is implemented in a work flow scenario, thus the users for production only need to upload via a portal their new data and parameters for the analysis. The users are informed about status and final plausibility result checks. The rest is shielded for security reasons. Currently, the ECG has a capacity of $\sim 15$ Tera FLOPS already available for user applications (total existing capacity: 20,000 desktop PCs, 330,000 computing cores, $\sim 50$ Tera FLOPS). This corresponds to a $\sim 30 \mathrm{M} €$ investment. In absolute terms this is also one of the largest dedicated computer resources world wide available to users via a central entry port managed by the Erasmus Computing Grid Office (ECGO). The ECGO is the secretariat in front of the technical infrastructure, supporting users, technical maintainers of the desktops, and serves as the development hub for grid as well as special user wishes. The aim of the ECG is to serve the areas of research, education, and diagnostics according to the mission of the donating public organizations. Beyond, the aim is to develop the ECG as a general broker organization for computing resources also for industry and other sectors. 
Therefore, the ECG is also connected to other grid and cloud infrastructures and respective European initiatives as e.g. the German MediGRID/D-Grid initiative, the European EGEE and EDGES infrastructures and several other local resources.

MediGRID (Krefting, 2008; Sax, 2007, 2008) and its services branch Services@MediGRID operate the national German biomedical research and care grid and is one of $\sim 20$ community grids of the German nation wide D-Grid initiative. The resources are cluster computers, which are located and maintained at local universities. Their size varies from 16 CPUs with 2 or 4 computing cores each (i.e. 32-64 cores) to 2048 CPUs with 4 cores (i.e. 8192 cores). These resources run different middlewares and can be accessed by the users via a central access portal or a central access to the resources directly (Fig. 2). Here again the userfriendliness is of major importance to gain a broad group of especially noncomputing experts. Special security protocols allow data transfer between the clusters under high-security medical conditions. Thus, the German MediGRID is said to be one of the most advanced health grids in the world combining data storage, computing power and sharing of applications in an entire nation. To serve the aims of research, education, and diagnostics in the biomedical research and care sectors MediGRID is organized in different modules, which are distributed via different institutions throughout Germany and thus form a more or less decentral organization. Nevertheless, special services, business modules and strategies were developed within the Services@MediGRID project allowing the grouping into different service classes and thus to apply different business and accounting models to distribute and organize appropriate the usage of the grid most efficiently. This also includes the possibility for billing and thus in principle commercial usage. Since MediGRID is located in the national research arena the latter is currently mostly valuable for accounting within the research community to balance and monitor the money flow within German research.

The Amazon EC2 cloud favours now an even more concentrated production facility since it exists of a few data centres around the world with massive cluster computing capacity of hundred thousands of computing cores at one centre. The centres are localized according to environmental and business aspects, i.e. that cheap energy supply for cooling, operation, and local subsidies are the main location factor despite a high capacity connection to the rest of the internet. The administration is done centrally in each facility, with different operating systems available and generic portals for user access. The centres are shielded entities and guaranty maximum security despite the country and legal setting they are in. Due to the size, users have access to a free scaling system, for which they are billed per computing hour on different accounting and business models. Amazon also helps to develop together with users their solution of interest, however, focuses mostly on providing pure hardware, the operating system and the access to the resource.

Obviously, the ECG belongs to the class of individual/public desktop grids with a pluristic, polytechnic and local/decentral approach, whereas the Amazon EC2 cloud is clearly industrial/private with a monopolistic, monotechnic, and central attitude. The German MediGRID and thus D-GRID is a mixture of both: government and public, not too pluristic, polytechnic, and local/decentral and neither industrial/private, nor monopolistic, monotechnic, and central. Consequently, this shows similar property and power structures as in the energy sectors including the current phenomenon to set up overcome giant monopolistic structures in the new cloud infrastructures, which are blocking fast development towards new more efficient opportunities. Thus, generically 


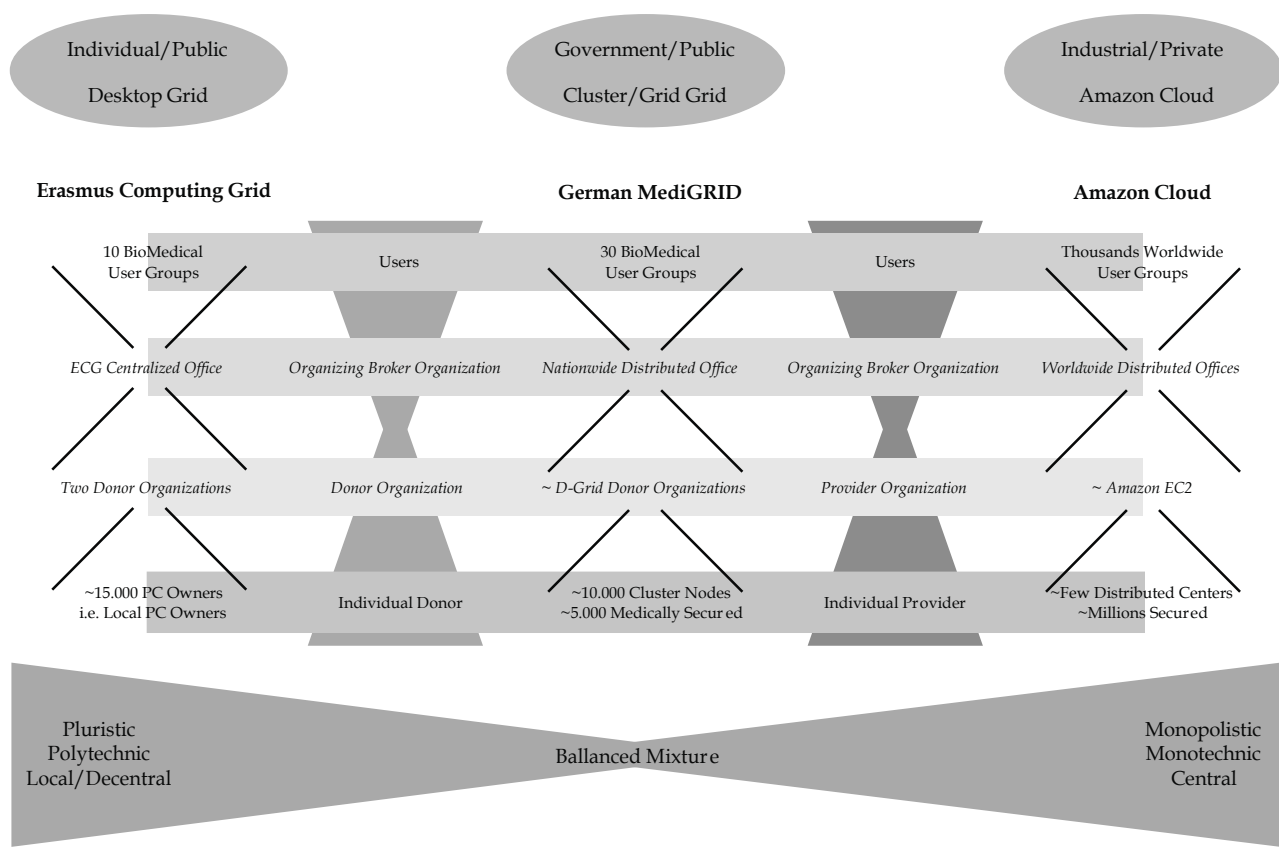

Fig. 2. Abstraction and detailed structure of the Erasmus Computing Grid, the German MediGRID, and the well known Amazon EC2 cloud. The three pillars are characterized by i) individual/public "private" grids, ii) government/public grids and iii) the very few industrial privately owned international clouds. Again all show the four levels involved in grid infrastructures: i) users, ii) organizing broker organizations, iii) donor organizations, and iv) individual donors. Again the three pillars are characterized by their means of capacity: i) small scale desktop and small mainframes, ii) regional and medium sized clusters, and iii) classic large scale cloud centres. And again whereas the first can be characterized by pluristic, polytechnic local/decentral means, the last is characterized by monopolistic, monotechnic and central terms. Although, the details may vary the structure leads to similar changes on the micro and macro level, which can be understood by the Human Ecology rectangle.

again four levels of organization are involved also in grid organizations: i) users, ii) organizing broker organizations, iii) donor organizations, and iv) individual donors. In a more abstract form this shows again that actually there are i) individuals and ii) societies of individuals, which are both involved on each of the organization levels, with a different degree of influence. Consequently, there is again a micro level from which a macro level emerges, with influence on the micro level, i.e. that both levels are connected in a complex and cyclical manner as in any evolutionary evolving system. Thus, the micro level is constituted by an invironment and the macro level creates an environment. This we will later see constitutes already again the Human Ecology rectangle as in the case of the energy sector. 


\section{Tragedy of the systemic complexity and the solution for a systemic sustained resource exploitation}

Primary energy or IT resource exploitation in reality involves a complex chain of events already on a technical level from the resource to usage, and involves e.g. resource i) acquisition, ii) basic exploitation, iii) storage and provision, iv) transformation to usable form, and v) transport to or access from users. Consequently, for a highly efficient exploitation of this chain, i.e. reaching a high efficiency from the primary resource to the end usage and thereby minimizing both the recyclable and unrecyclable losses, these five steps have to be optimized in a systemic manner to guaranty a careful exploitation of the primary resource. Optimization means here that not only the different steps are optimized within themselves but beyond, that the overall efficiency of the chain is optimized, since the optimum in on step of the chain might as a side effect reduce even the level of efficiency in another step or the rest of the chain far below the optima of these single steps and thus reduce the overall efficiency of the entire exploitation chain. Thus, the exploitation chain is already a complex system, where different parts influence all other parts.

It is obvious that usually the chain is seen mostly from each end, i.e. that the perception of the exploitation chain is either i) resource oriented or ii) usage oriented: i) it is easy to look only on the energy or IT resource and then think about its exploitation without looking at the usage, and ii) it is equally easy to take only the perspective of the usage and user and neglect the meaning for the primary resource exploitation. The first perspective is often taken e.g. in fossil energy business models where power plants have at the beginning been built near the coalmines. Equally, big hydroelectric dams can only been built locally whether there is a usage at hand or not. Often then potential users take the opportunity to move to that location. The second perspective, however, is often taken due to the low transport costs of fossil resources, i.e. coal is just transported around the world to the user. In contrast, renewable energy or grid IT approaches try to combine both perspectives: here first the two questions are asked at the same time: i) what demand exist locally, and ii) what resources are available locally? If availability and demand cannot be brought in agreement locally then the radius can be broadened from local to regional and so forth. In either case then the details of the different steps have to be questioned and the best mix of resources - if there are different ones - has to be considered, thus the whole exploitation chain can be optimized. This is, of course, a dynamic process, i.e. the optimized exploitation chain will most likely vary over time, i.e. that the time factor of a technology replacement or entire new introduction has also to be considered. In the case of renewables this involves less the primary resource, which is one of the biggest advantages of renewables and leads already to a sustained availability of the primary resource, which nevertheless is only one chain part.

The complex exploitation chain is, however, still a too simple rationalization, since the chain from the resource to usage involves on each of the five described technical levels again other chains of events, which need again various resources i) energy, ii) information, and iii) materials. Thus, respectively, the exploitation chain itself needs to exploit energy, information and material resources to exploit the resource of interest, as e.g. energy or IT resources. Consequently, this means that the exploitation chain is in reality a complex network of exploitation chains based on the availability of the same and other resources. And on these secondary levels again exploitation sub-chains exist and so forth and so forth... Thus, an exploitation network makes naturally the situation tremendously more complex then just a complex exploitation chain, not only because there are even more 
components to be considered, but mostly because the number of non-linear interactions of these components are even higher, more complex, and more dependent on each other. Especially the recursive dependence on the primary exploitation chain by the secondary, i.e. that also the secondary exploitation chains nested on each of the primary exploitation chain levels, need e.g. energy or IT resources to be able to function, is here of major importance in terms of complexity increase. The influence is now not only non-linear but also adds many an exponential interaction, which can in- or decrease the importance of a small tiny factor somewhere in the exploitation network enormously.

Beyond, an exploitation network connected to a specific e.g. energy or IT resource now involves many other resources, which have as well their limits and renewability aspects. Especially, material resources play here a fundamental role as - additional to energy and information resources - e.g. steel for the primary resource exploitation within machines and transport systems, rare metals for the transformation as e.g. metal catalysts or the dotation of the silicium in photovoltaic cells as well as copper for energy or information transmission or lithium for high capacity storage energy, to name only a few. Consequently, the whole resource and exploitation sector already on the technical level is a multi-recursive network of resources and their exploitations, where finally the single small component, its resources status, and renewability, might be as important as the major resource to be exploited. Nevertheless, there is a caveat in this on first sight depressing and seemingly unsolvable complexity. There is a natural hierarchy of the importance of and the amount of resources: If a resource is renewable or at least highly recyclable then these limits are tameable as well as in the case of resource replacement. Whereas for a fundamental resource like energy or information this can be tremendously difficult, for the materialistic part in exploitation networks that might in most cases be possible due to the fact that with the building box nature provides us in the many physical elements and thus the chemical products one can make thereof. So it is possible nowadays to replace steel by carbon fibre, i.e. heavy industry products by light chemical materials. The same holds for many components, although all follow again the resource limit, exploitation network, and renewable or recycling generic challenges as already discussed.

Consequently, for a highly efficient exploitation of such a complex systemic network, i.e. reaching a high efficiency from the primary resource to usage, all single steps have to be optimized in a systemic manner to guaranty a careful exploitation of the primary and all other involved resources. Actually the exploitation network becomes now also a general resource network. Thus, optimization means here that not only the different steps are optimized within themselves but beyond, that the overall efficiency of the entire network is optimized, since the optimum in one step might as a side effect reduce even the level of efficiency in another step or the rest of the network far below optima of these single steps and thus reduce the overall efficiency of the entire resource and exploitation network. Thus, the resource and exploitation network is already a hyper complex system, where different parts influence all other parts, which creates in principle what can be termed the Tragedy of Systemic Complexity:

\section{The Tragedy of the Systemic Complexity:}

Resource exploitation is a complex systemic network with a huge number of technical components and beyond huge exponential non-linear interactions between these components,

thus highest efficiency can only be achieved by a systemic optimization with contradictions appearing on the level of single components

leading to huge individual and social paradoxical challenges of perceptions and understandings. 
This now has huge consequences for the implementation of a highly systemic exploitation network by individuals and society, since now all individual components have to be optimized themselves with respect to all other components and the complete complex hyper systemic exploitation network. Therefore, the classic reductionistic approach is unavoidable as long as it finally ends in a holistically reintegrated systemic result. Consequently, for a highly efficient energy and IT resource exploitation this is the vital core to Sustained Renewability, since only then also primary renewable resources are not compromised by the limits in the complex resource and exploitation network and since only then enough resources will on human time scales always be available to exploit this primary resource and thus sustain its exploitation and usage for again on human time scales indefinite time. Thus, the technical level requires a holistic systemic approach for Sustained Renewability.

\section{The inverse tragedy of the commons in the renewable energy and grid IT sectors}

Analysing both the energy and IT resources, their means of exploitation and usage scenarios in a systemic manner, clearly shows beyond a pure estimate of scale, that obviously there are affluent resources and also solutions for their efficient exploitation available. The Tragedy of the Systemic Complexity thereby shows that already the pure technical means in the entire chain from the resource to usage has to be taken special care of, since otherwise the loses are far too big, i.e. the efficiency is far too low. E.g. considering overall efficiencies below $1 \%$ would mean that a system of resources would be 100 times faster depleted than in the, of course, impossible case of $100 \%$ efficiency. Again it should be stressed that this must include beyond the basic energy or IT resource themselves, their entire exploitation chain and thus the resources needed for this exploitation as well. Renewables and grid are means of doing that in a very sophisticated manner at least to a larger extent then the classic large-scale power plants, computing centres, or cloud infrastructures. Thus, the fundamental basis for Sustained Renewability means solving the technical systemic complexity in a productive manner, i.e. the technical chain from the resource to usage must be exploited in a systemic manner already on the pure technical level. Without such a systemic approach for resource exploitation, no Sustained Renewability can be reached. Thus also all the limits have to be considered in the entire exploitation network, since even if there is a huge resource their might be a tiny but nevertheless bottleneck within a resource network as e.g. a rare metal or substance which is crucially needed somewhere in a corner of the exploitation network. Nevertheless, exploiting the renewable energy or IT resources in a systemic approach purely technical, i.e. by integrated holistic ecology like systems e.g. by using the renewable resource or sharing the available IT infrastructure, seems hard since both renewable energy and grid infrastructures slowly emerge, which is due to their importance and especially in the IT sector with its great opportunities and fast turnover rates, paradoxical.

The analysis of energy and grid organizations showed already generically that there is a micro level from which a macro level emerges. Actually, the organizational chain suggests that this is true for every resource exploitation network and thus is its fundamental cultural root, especially if the resources, which are to be exploited, are part of the commons as the renewable energy (i.e. in the climate change dilemma) or IT resources (i.e. the dilemma of e.g. to little information transforming capacities) in principle are. Both this micro and macro level now wraps the technical exploitation into the dialectics from invironment to environment. In the case of over-exploitation of common resources, the exactly same 
complex interplay between the individual and the society as well as the invironment and the environment appears. This his is well known as the so called Classic Tragedy of the Commons (Hardin, 1968, 1994, 1998; Ostrom, 1990; IASC; Commons), in which (multiple) independently acting individuals due to their own self-interest can ultimately destroy a shared limited resource even though it is clear that it is not in the long term interest of the local community and society as a whole. The phenomenon in principle is nothing new and appears already in ancient myth and religions, since it concerns the basis of life, i.e. the usage of resources in general and in particular that of energy and food. The violation of the carrying capacity, the theft of resources connected with the decline or death of those who lose their resource basis, and the squandering of resources are deeply integrated in all cultures reaching the level of archetypical traits. E.g. the Irish measure for the size of a piece of land in callop's describes the size in terms of the carrying capacity for different animals: the same sized piece of land, i.e. e.g. one callop might be 10 cows, 12 horses, or 40 sheep. In the case of under exploited resources there seems to be the same phenomenology of the Classic Tragedy of the Commons challenge put forward. This phenomenon is now theoretically defined - as a logical consequence - as the Inverse Tragedy of the Commons (Fig. 3):

The (Classic) Tragedy of the Commons:

A resource belonging to all and being on limited demand is

OVEREXPLOITED by the user due to responsibility diffusion!

$$
<=>\text { TRANSFORMATION }<=>
$$

:The INVERSE Tragedy of the Commons

A resource belonging to all and being in affluent availability on limited demand is UNDEREXPLOITEDby potential users due to responsibility diffusion!

Interestingly, not only is responsibility diffusion the most likely and general reason for the appearance of both tragedies, but also the psychological description for both the micro and the macro level hits the same archetypical traits (Fig. 3) as mentioned above. Whereas the Classic Tragedy of the Commons corresponds to i) indifferent hedonists, ii) careless players, and iii) the chronic overstrained, the Inverse Tragedy of the Commons corresponds to i) hedonists, ii) the cool calculating, and iii) the tragic hopeless. The under-used potentials, i.e. the general basis (matter, energy, information, biological, psychological, and societal level) and over-used resources (materials, reservoirs, memes, organisms, behaviours, cultures), clearly show how from a virgin resource opportunity, concrete objects emerge with their attached limitation burden (Fig. 3). Nevertheless, they all cluster around a systemic technical development. Thus, the complex field created, describes exactly the tension found in resource limitation phenomena and evolutionary emergence.

Consequently, it becomes now also clear what constitutes the micro and macro levels: on the micro-social level the systemic and the open sharing attitude of the individual play the key roles, whereas on the macro-social level the organization culture of the embedding institutions and in the end society as a whole as well as its cultures are the central points. I.e. that beyond the technical implementation of an exploitation network in a systemic technical manner, but nevertheless still technically focused approach, the individual and society with their invironment and environment they consist of and create respectively, need to be considered as the crucial to be investigated fields to understand why still with even a systemic technical exploitation scheme with highest efficiencies existing, even the best solved Tragedy of Systemic Complexity is not implemented (not to speak of the development 
Inverse Tragedy of the Commons

hedonists

cool calculating

tragic hopeless
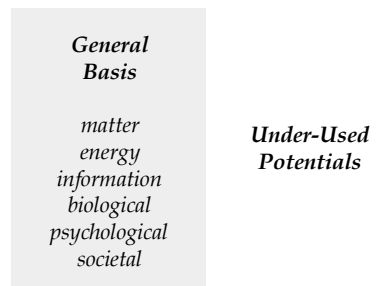

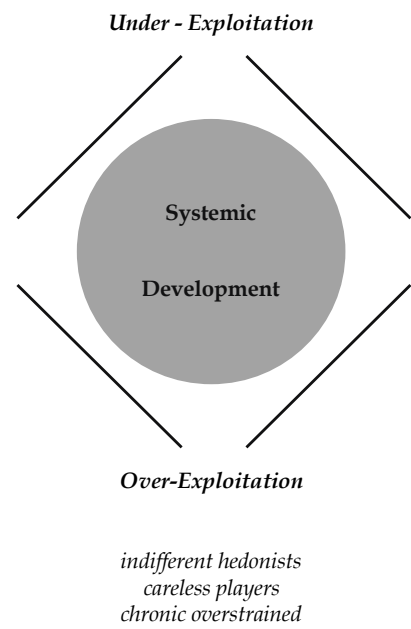

Classic Tragedy of the Commons

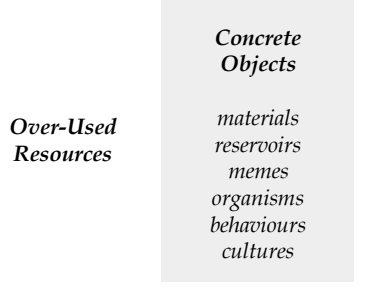

Concrete

Cojects

memes

organisms cultures

Fig. 3. Generalization of the Inverse Tragedy of the Commons: the Classic and Inverse Tragedy of the Commons are directly complementary to each other and can on the deep psychology level be associated with complementary pears of archetypical behavioural traits. This is in line with the complementary under-used potentials and over-used resources, which emerge from the potentials by freezing of potentials into concrete objects with corresponding limits. Both correspond to the general basis and concrete objects. Whereas the Classic Tragedy of the Commons corresponds to i) indifferent hedonists, ii) careless players, and iii) the chronic overstrained, the Inverse Tragedy of the Commons corresponds to i) hedonists, ii) the cool calculating, and iii) the tragic hopeless. All cluster around a systemic technical development as the technical core.

of a solution for the Tragedy of Systemic Complexity). Thus, the system of i) technical system, ii) micro level, and iii) macro level has now to be investigated as a system in a holistic systemic manner itself. Therefore, pinpointing the phenomenon of under exploited potentials to the same phenomenological root as the well known phenomenon of over exploited resources opens now the complete opportunities and tool set to examine the challenge of introducing a Sustained Renewability approach into practice (as e.g. in the renewable energy or grid IT sectors) as well as its principle day-to-day management. Thus, practical implications can be derived from an analysis of the micro and macro levels, which then have to be embedded in a systemic manner and approached on a theoretic level. Appliance of this concept with practical guidelines, the implementation of Sustained Renewability practically, can be realized in principle for every resource management sector. 


\section{The tragedy of autopoietic social subsystems}

The challenge to integrate exploitation measures of energy or IT resources, which follow technically a systemic approach and thus beat the Tragedy of Systemic Complexity, into society involves naturally all stakeholders of society (Fig. 1, 2). The existence of a Tragedy of Systemic Complexity and an Inverse Tragedy of the Commons and its macro social aspects, point to the major importance of the interaction complexity of the social subsystems theory by Niklas Luhmann (Luhmann, 2004, 2008), i.e. a systemic approach analysing and describing the social system and the subsystems it consists of: It is based on the autopoietic concept of Humberto Maturana and Francisco Varela (Maturana \& Varela, 1992), and is the most advanced social systems theory, describing the huge complexity of the macro sociality of the renewable energy as well as grid IT phenomenon. An autopoietic system is a network of processes consisting of: i) interactions and transformations continuously regenerating and realizing its networks of existence, and ii) the constitution of the system as a unity in space in which the component exist by specifying the topological domain of its realization. Central to this description of evolutionary emergence, i.e. self-reproducing systems, is the material and information exchange between the components. Social systems are obviously communication systems, with society being the most encompassing one. Immediately that makes clear what challenge that suggests: what are the social systems, is there more than one, if so how do they interact, and most importantly who can they act together in a systemic manner to achieve a goal which is either emerging from one system internally or introduced from the outside. Consequently, many of the conundrums appearing during the society internalization become evident and are in agreement with the Tragedy of Systemic Complexity and the Inverse Tragedy of the Renewable and Grid Commons:

Around seven social subsystems can be defined and reflect the evolutionary emergence from deep psychology to society (Luhmann, 2008): i) religion, ii) education, iii) science, iv) art, v) economy, vi) jurisdiction, and vii) policy. All of these systems have their internal code of communication and their own connectivity interface to the other subsystems. Thus, the Tragedy of Systemic Complexity struck now in principle again, although this time on a social level, which results in huge barriers: e.g. the religious code of believe or notbelieve is incompatible with the have or not have money code of the economic sector. This is even truer for science (true vs. non-true), jurisdiction (just vs. un-just) and politics (power vs. no-power), which have nothing to do with education (knowledge vs. noknowledge). Renewable energy exploitation belongs to several subsystems, mainly those of science and economy in contrast to grid IT infrastructures, which belong currently mostly to the academic sector (de Zeeuw et al., 2007; Krefting et al. 2008; Sax et al., 2007, 2008). Despite the success of both renewable energy exploitation approaches as well as the widespread usage of grid IT infrastructures within society, the broad rollout, i.e. the internalization of systemic approaches into society, is decelerated by the lack of interoperability between these subsystems. Consequently, the Inverse Tragedy of the Commons results in

\section{The Tragedy of Autopoietic Social Subsystems:}

Subsystems have their own code of communication and are separated from each other in a way blocking in principle a consistent integration although they form a society, with all their contradictions, which thus leads to blockage of the system. 
This macro level tragedy clarifies that renewable energy and grid IT organizations are just another example for complex infrastructures whose efficiency increase depends beyond more or less complex technical solutions on the participation of all subsystems concerning their societal internalization. In detail this means that each of those social subsystems must be analysed according to their internal constituents in respect towards the implementation of a systemic approach in respect to the status quo as well as to the ability to react to an until then not used or entire novel systemic approach. Thus, it might be, that such a systemic approach might not at all be implementable within such a subsystem at first, that major transformation need to be made, or that in the best case already existing structures can be used. The same holds for the communication between the subsystems, since here different internal preparedness levels might either ease or worse the communication in respect to such an implementation. Consequently, the challenge of implementation of Sustained Renewability approaches into society involves again two levels: On the micro level of individual subsystems the move towards implementation depends on the subsystem "stickiness" of individuals. On the macro subsystem level the integration of institutionalized subsystems via soft interfaces, which allow the communication barriers to be lowered, is central. Both has to be taken care of since this is given beyond the systemic pathways within the subsystem and the setting how subsystems can be moved or interact with others.

The acceptance of this is an important knowledge opening huge opportunities to examine and approach the challenge of introducing renewables or grids and their management. Beyond, this clarifies the challenges in all other exploitation sectors (probably residing in other subsystems) since all subsystems should always be involved. Thus, the Tragedy of the Systemic Complexity in terms of systemic integration into society can be understood and has to be taken into account. Beyond, the Classic and Inverse Tragedy of the Commons are a societal challenge with the opportunity to be resolved, if as well the technical systemic approach is combined on the social system level in a sustainable systemic manner.

\section{The tragedy of security/risk/profit psychology}

Since the macro level of social subsystems emerges evolutionarily from the micro level (Egger, 2008), one needs to consider the individual for whom each implementation and internalization of a new technology is based on a positive relation between the risk and the profit involved from the perspective of the individual. This is the core of any action a human individual takes and defines the degree of motivation a person commits to an action, i.e. the change of something in contrast to doing nothing. Thus, the level of altruism leading to successful implementation as in the renewable energy case or the sharing in the grid IT case on the individual level and its commitment beyond its own job/agenda, as well as that of its own institution without incentive structure to take responsibilities, is essential and leads to responsibility diffusion: Even the obvious winwin situation renewables like photovoltaic on the roof, or small hydroelectrics on the grounds of an individual, are hard to communicate and even the clear effects of producing with no energy resource costs the own energy result in slow implementation of renewables compared to the benefits and the climate issue at face. Even more so, the clear win-win situations for individual grid users are under these circumstances hard to communicate and even the additional networking effects result hardly in the set-up or usage of grids. It is also unlikely that people take the risk to exceed their own budget and corresponding responsibilities, when future results and its benefits are unclear to them. 
As long as exploiting the renewable energy resources or sharing in the grid IT sector is voluntary and in hand with uncertainty and risks, it is less likely that individuals will behave altruistically on behalf the societal benefit. Consequently, on the micro level the situation is that of a perverse Inverse Tragedy of the Commons: the commons is not abused or overexploited, but in contrast the tremendous resources are not used at all despite the needs and obvious benefits, due to secondary (mostly "irrational") interests.

Thus, the integration challenge involves the individual of the different institutionalized society stakeholders in a very deep way since these individuals shape the individual actions according to their function in a social subsystem. How an individual perceives the security/risk/profit ratio depends on its personal security/risk/profit psychology matrix:

Deep Psychology Security/Risk/Profit Cascade:

emotional individual $s / r / p$ perception

rational $s / r / p$ knowledge acceptance

internalized incidental $s / r / p$ behaviour

accepted legal and political $s / r / p$ scenarios

lived religious and cultural $s / r / p$ archetypi
:Autopoietic Subsystem Correspondence

genetics and deep psychology education and science economics

jurisdiction and politics religion, art and culture

Thus, this matrix describes a similar challenge on the micro level similar to the macro level with conflicting personal positions and internal balancing the invironment with the environment. This creates on the micro level again a tragedy:

The Tragedy of Security/Risk/Profit Psychology:

Individuals balance constantly a complex combination of invironmental and environmental security/risk/profit deep psychology factors, whose contradictions lead to responsibility diffusion.

In detail this means that each of those levels need to be considered especially from key individuals, i.e. of those, who hold important positions within social subsystems, to just the collective invironment of an entire population. And again this poses two obvious challenges in a systematic concept: on the micro level, the risk perception and the emotional well-being of the individual has to be considered, whereas on the macro level, the risk perception in the procedural and institutionalization in organizations have to be considered, i.e. the interaction of the individual with the organization it is working in. Thus, it might be that such a systemic approach might not at all be implementable with certain individuals or collective emotions in place at first, that major transformations need to be made, or that in the best case already existing structures can be used. Unfortunately, the identification and analysis of this tragedy is by far more challenging in every respect and especially concerning management guidelines, due to the hardly changeable basis, due to its genetic and evolutionary basis and the time scales involved to change archetypical societal concepts, in contrast to the macro level, where bypassing measures and changes can in principle be implemented at will, i.e. major screws can be relatively easy adjusted by order with or without societal participation and/or agreement. Consequently, this tragedy has to be tackled with big care and shows that the Tragedy of Systemic Complexity as well as that of the Inverse Tragedy of the Commons can really be addressed by a Human Ecology rectangle approach integrating the different tragedies in a systemic manner and thus to reach systemic renewability by such super-systemic approach, as will be shown in the following. 


\section{Human ecology for a sustained renewable energy and grid IT resource network exploitation}

To overcome the Tragedy of Systems Complexity and the Inverse Tragedy of the Commons together with the base tragedies of the latter, the Tragedy of Autopoietic Social Subsystems as well as the Tragedy of Security/Risk/Profit Psychology in the renewable energy and grid IT sectors a systemic approach on the technical level combined with an approach to tackle the micro and macro social levels is crucial to reach Sustained Renewability in these and in principle generically any exploitation sector. The basis of all these is the level of complexity in the corresponding areas leading to the heart of the matter - responsibility diffusion. For this the inter- and transdisciplinary field of Human Ecology (Egger, 1992, 1996, 2004; Bruckmeier \& Serbser, 2008) gives a framework for their combination, followed by understanding and approaching direct guidelines for the management of renewable energy and grid IT resources. I.e. Human Ecology embeds the technical systemic solutions with a systemic approach on the micro and macro level of societies. Human Ecology was developed originally by Robert Park (1864-1944) and Ernest Burgess (1886-1966) and evolved in Chicago in the 1920's in close connection to the field of city development. Here complex questions and challenges arose, ranging from e.g. i) fundamental technical questions of how to structure a city in terms of spatial use, transport of the basic supplies as energy and water, and the removal of waste, ii) of how to structure and organize social needs from governmental services and schools to commercial shopping malls to economic entities for production, as well as iii) cultural issues as how to plan a modern human city which allows everybody to achieve a fair share of the pursuit of happiness, whether one belonged to the poor or the wealthy part of society. By analysing the different stakeholders playing the fundamental roles there, the complex system challenges appearing were abstracted on the social level, since this was seen as the main issue of the - at that time - not yet in detail defined and worked out Tragedy of the Commons. Thus, Human Ecology classically deals with the complex interplay between i) the individual, ii) the society, and iii) the environment, which usually is symbolized in the so called Human Ecology triangle. This triangle is the paper tool representation and believed to be the core of the complex interplay factors in society. The framework has been used to investigate many a complex mankind related challenges as e.g. the exponential demand growth until reaching a limit, its inherent property of life and evolution, as well as waste and pollution related issues, i.e. in principle all the above mentioned tragedies. Obviously, these sustainability questions beyond the materialistic world are found on all evolutionary levels up to the psychological, societal and cultural one and involve also every cause for exponential growth, which is the major reason for reaching the natural unchangeable and thus unavoidable limits extremely fast.

Already, the Tragedy of the Systemic Complexity on the technical level shows that this rationalization and projection to three major constituents needs at least to be extended by a systemic approach on the technical level or better, the technical systemic approach must be embedded within the triangle. Beyond, the detailed analysis of the generic organization of the fossil and renewable energy as well as grid and cloud IT infrastructures proposed a micro and a macro level. Thus additionally, the detailed dissection of the Inverse Tragedy of the Commons by investigating the Tragedy of Autopoietic Social Subsystems and the Tragedy of Security/Risk/Profit Psychology, proposes the extension of the classical Human Ecology triangle to a rectangle consisting of: i) invironment, ii) individual, iii) society, and iv) environment 
(Fig. 4). Consequently, here the invironment is added, since it is the core on which the individual is based or in other terms, due to the presence of the irrational part of the individual in respect to its security/risk/profit psychology, the latter can also be accounted for. Thus, the Human Ecology rectangle describes the relation between the invironment (Innenwelt), the individual, the society and the environment (Umwelt). The invironment and the environment as well as the individual and the society are complementary pairs and create a field. The invironment thereby constitutes the Innenwelt, the individual forms society, the individual society creates an environment as the invironment constitutes much of the society. And consequently, the rectangle reflects the micro level (invironment and individual) and the macro level (society and environment) correctly, or in retrospect the micro and macro level constitute each half of the Human Ecology rectangle. This fits the field of the Classic and Inverse Tragedy of the Commons, with its under-used potentials and overused resources, i.e. means that both can be correspondingly overlaid and connections can be made accordingly. Consequently, the invironment is the missing link to reach systemic completeness of Human Ecology, and thus round it up to its full power in terms of usability concerning the management of systemic challenges as put forward by the renewable and grid IT challenges on a practical level. That means that the Tragedy of Autopoietic Social Subsystems as well as the Tragedy of Security/Risk/Profit Psychology which are the heart of the Classic and Inverse Tragedy of the Commons can not only be wrapped in a systemic framework which is complete in its constituents, but moreover, that this framework now can be really applied to the solution of the Classic and Inverse Tragedy of the Commons much better then with the Human Ecology triangle alone. This is important since without such a systemic framework and the internalized knowledge always perceived paradoxes will appear, which cannot be understood and thus cannot be resolved adequately on the level required. To reach its full power also in respect to the Tragedy of the Systemic Complexity on the technical level, additionally, now this needs to be extended again by a systemic approach on the technical level or better, the systemic approach must be embedded within the rectangle again, since without this technical level the complete system of technology, micro and macro level would again be not complete. Now this means nothing else than that the complete system of technical development and implementation has to be considered as well as the security/risk/profit psychology of the individual with its invironment and the autopoietic subsystem organization of society with its environment. On first sight this insight to take a holistic viewpoint and make that the basis for solving the issues involved with the renewable energy, grid IT or any other complex exploitation network seems natural and in principle is completely obvious - actually not even be worth thinking about. However, the fundamental issues and challenges faced in exploitation networks to be implemented to reach Sustained Renewability, i.e. to solve the problems of resource network limitations and thus to overcome the fundamental limits of energetic and material consumption growth reaching carrying capacity limits by the classic approach, are obviously there and demand urgent solutions in respect to the urge of the problems involved if nothing substantial is changed. Thus, the pure existence of the climate challenge shows the importance of a Sustained Renewability approach which overcomes the technical Tragedy of Systemic Complexity and the Inverse Tragedy of the Commons, in which resources are not unsustainably overexploited but in contrast used in a Sustained Renewability way holistically integrating the i) technical resource exploitation networks and ii) all the autopoietic social subsystems on a macro level as well as the psychology of individuals on the micro level, in an holistically 


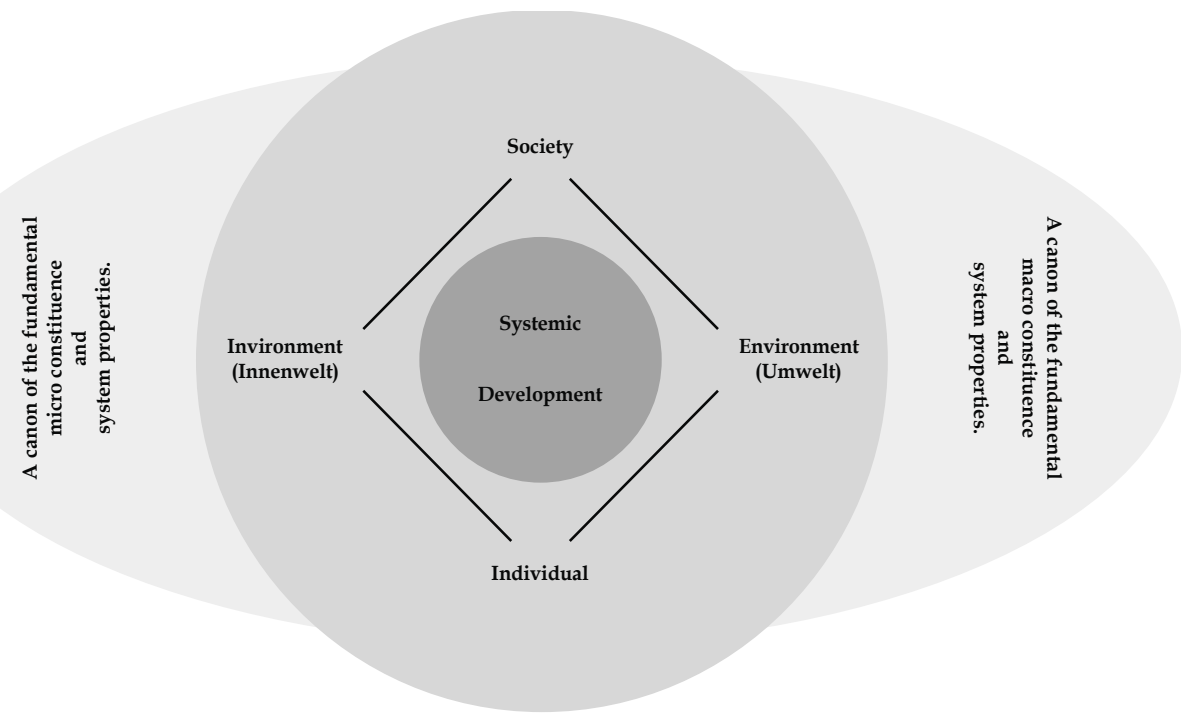

Fig. 4. The Human Ecology rectangle describes the relation between the invironment (Innenwelt), the individual, the society, and the environment (Umwelt). It is the extension of the incomplete classical Human Ecology triangle, which consists only of the individual, the society, and the environment but not the invironment. The invironment and the environment as well as the individual and the society are complementary pairs spanning a field between them. The invironment thereby constitutes the Innenwelt, the individual forms society, to the individual society gets a general environment as the invironment constitutes much of the society. Within the rectangle on each level the systemic aspects of each system and development thereof need to be encountered. To handle the invironment and the environment a canon of their micro and macro constituents and system properties is necessary.

systemic ecology like manner. Consequently, Sustained Renewability can be fundamentally defined according to the most fundamental and classic definition of the classic (biogene) ecology (Haeckel, 1866, 1898; Knoch, 2009, 2010): 
The Definition of Sustained Renewability and thus the Combination of Technical Systemic Theory with Human Ecology:

"Under Sustained Renewability, i.e. Technical Systemic Human Ecology, we understand the complete science of the relationships of Sustained Renewability to the surrounding environment to which we can count all conditions of existence in the widest sense."

(Sustained Renewability is)... the relationship between the technical system complexity and all micro/macro constituents of Human Ecology."

\section{Sustained renewability by systemic theory and human ecology means}

Without doubt both the growth of the world population and the ever-new technologies emerging from R\&D - both creating ever higher needs as well as expectations - also the energy and information amount to be acquired, stored, transformed, and finally used is exponentially growing and due to the classic reductionist approach reaching the fundamental limits and due to pollution also the carrying capacity of earth. Nevertheless, it is also obvious that there are huge renewable energy and grid IT resources available as in most other resource networks, concerning technical production or any social level. This results in many opportunities of which most, however, are not realized, i.e. introduced and internalized into society. In contrast, ever more resources are said to be required but believed to be at their limit and thus already unavailable for further exploitation. Especially in the energy and IT sector the demand still grows exponentially and is satisfied still with antiquated solutions. Although exponential growth inevitably will lead to limits sooner or later, there seems to be also many an opportunity to sustainably manage resources on very long time scales. Thus, clever resource management can increase the efficiency tremendously and in consequence avoid limiting barriers as e.g. integrated chemical production or sophisticated agro-forestry systems show. Renewable energy and grid IT infrastructures are believed to be such solutions which exploit under-used and available resources by a Systemic Renewability approach, which in principle is based on a simple holistic systemic analysis of the technical systemic complexity combined with a Human Ecology approach. Both have in common that their technological turnover rates are faster than in classic dinosaur approaches, although especially the grid IT sector with its fast technological turnover rates, however, allows to bring innovation opportunities very fast to the market and thereby increase the efficiency from the resource to usage tremendously. This could results on the one hand in lower investments into infrastructure, which obviously would provoke large resistance by the producing industry, or on the other hand results in a much higher output and thus return of investment made by society in these infrastructures, which would give a big "present" with only minor further investment to society as a whole. Nevertheless, it remains a big issue despite all the efforts of the renewable, the grid IT, and any other resource exploitation sector, why the obvious huge benefits of much higher resource exploitation network efficiencies is so hard to internalize into societies despite its crystal clear benefits concerning the fundamental limits of resource exploitation, carrying capacities as well as economic, social and cultural benefits already in the short but even much more so in the long term.

The Tragedy of the Systemic Complexity shows already what complex exploitation networks mean, since in reality a complex network of resources and exploitation based on the availability of the same and other resources exist instead of a nevertheless complex 
exploitation chain. Thus, an exploitation network makes naturally the situation tremendously more complex than just a complex exploitation chain due to the number of i) non-linear, ii) nested, and thus in the end iii) exponentially recursively linked interactions of these components since they are even higher, more complex, and more dependent on each other. Especially the recursive dependence on the primary exploitation chain by the secondary, i.e. that also the secondary exploitation chains nested on each of the primary exploitation chain levels need e.g. energy or IT resources to be able to function, is here the main driver of complexity increase. The influence is now not only non-linear but also adds many an exponential interaction, which can in- or decrease the importance of a small tiny factor somewhere in the exploitation network enormously. Consequently, for a highly efficient exploitation of such networks, all single steps have to be optimized in a systemic manner to guaranty a careful exploitation of the primary and all other involved resources. Actually the exploitation network becomes now also a general resource network. This has huge consequences for the implementation of a highly systemic exploitation network by individuals and society, since all individual components have to be optimized themselves with respect to all other components as well as the complete complex hyper systemic exploitation network. Therefore, the classic reductionistic approach is unavoidable as long as it finally ends in a holistically reintegrated systemic result. Consequently, for a highly efficient energy and IT resource exploitation this is the vital core of Sustained Renewability, since only then also primary renewable resources are not compromised by the limits and since only then enough resources will be - on human time scales - always be available to exploit this primary resource and thus sustain its exploitation. Thus, the technical level requires a holistic systemic approach for Sustained Renewability.

Beyond, the organizational architecture analysis of renewable energy and grid IT infrastructures as well as their management shows that there are four levels of stake-holders involved: i) users, ii) organizing broker organizations, iii) producer or provider organizations, and iv) individual producers or providers. That is a much more complicated than the integrated production in chemical industry, since here one has a large spatial and cultural coverage in contrast to the "internal" situation of one single company. There is also a big difference to sophisticated agro-forestry systems as e.g. those in Indonesia, since these systems had a huge temporal time span for development. Although they involve in principle the entire society, the decisions are still taken by the single farmer and community despite their a posteriori internalization in tradition and cultural rules. Abstraction of the four levels involved in grid infrastructures leads to a micro level from which a macro level emerges, having again an influence on the micro level and vice versa. The micro level is constituted by an invironment and the macro level creates an environment, which already constitutes the Human Ecology rectangle as was shown. Consequently, here from the pure theoretical viewpoint not only complete consistency was reached proving the validity of the arguments, but also access to a "tool box" was gained to be used successfully for complex internalization issues. This is important for generalization and for justification of the thereof derived management measures. Beyond, the fact that renewable and grid IT resources are completely underused attributes to the phenomenon of the Inverse Tragedy of the Commons, i.e. that the resources are not overexploited unsustainably until their destruction. Together with the finding of the micro and macro level in the organization of grid organizations which plays the important role in both the Classic and Inverse Tragedy of the Commons lead to 
responsibility diffusion and thus inefficient resource management. This, consequently, makes clear that the grid challenge concerning implementation and integration of grids lies in the social embedding of the micro and macro level phenomena: i) the attitude/socialisation based on the security/risk/profit psychology of the individual, and ii) the culture of the embedding institution and society based on the interaction of the autopoietic social subsystems. This is similar for the renewables as well as the grid IT sector and also has huge implications for any other complex resource exploitation network sector. Considering the macro level more in detail reveals that the autopoietic subsystems theory describes the social environment best. Unfortunately, the social subsystems i) religion, ii) education, iii) science, iv) art, v) economy, vi) jurisdiction, and vii) policy, have a more or less incompatible code of communication which leads to the Tragedy of the Autopoietic Social Subsystems and thus to large inconsistencies and blockings. Consequently, the challenge lies in the integration of autopoietic subsystems towards a working society on the micro and macro level by i) approaching the subsystem stickiness of individuals, and ii) the soft bridging of subsystems. In the daily work individuals have first to realize their own working and borders of their and other social subsystems. In a second step the possible bridges between social subsystems need to be realized and concrete ways to circumvent inherent blockings have to be explored to reach the level of a joined effort realization. On the micro level the security/risk/profit psychology matrix plays the major role since for the individual each internalization of a new technology is based on a positive relation between the security/risk/profit involved. Even for clear win-win situations the phenomenon of responsibility diffusion can appear. Since individuals have to balance constantly between the invironment and the environment, i.e. between psychology and social subsystems, there appears also a hard to tackle Tragedy of the Security/Risk/Profit Psychology. Consequently, the challenge on the micro and macro level are given by i) the individual perception and the individual well being, and ii) the procedural and institutionalized careful management. I.e. for the daily work that the individual need to rationalize its own behavioural background and invironmental constituency, and that institutions need to accept and develop the invironment of their employees as well as the psychological status of the environment they create. Thus, the creation of awareness might not change the individual but by team formation with different characters and corresponding procedures, the openness in an institutionalized form can increase the internalization of new technologies.

Consequently, to overcome the challenges put forward by the Tragedy of the Systems Complexity, and the Classic and Inverse Tragedy of the Commons with its base tragedies, the Tragedy of Autopoietic Social Subsystems and the Tragedy of Security/Risk/Profit Psychology in the renewable energy and grid IT sector, a Sustained Renewability approach in a holistic ecology-like manner combining the micro and macro level is crucial for in principle every resource and exploitation network. The interdisciplinary field of Human Ecology gives a framework also for the understanding and approaching of the Classic and Inverse Tragedy of the Commons for direct guidelines in the day-to-day management of grids as well as other areas and combine the above into a unified framework. Theoretically, the analysis carried out here showed that it is necessary to extent the classical Human Ecology triangle to a rectangle with i) the invironment ii) the individual, iii) the society, and iv) the environment. Thus, from the pure theoretical viewpoint, not only complete consistency was reached proving the validity of the arguments, but also access to a "tool box" - which has been used already successfully - was gained. This is important for generalization and justification of 
thereof derived management measures. To reach its full power also in respect to the Tragedy of the Systemic Complexity this needs to also embed a systemic approach on the technical level, since without this technical level the complete system of technology, micro and macro level would again be not complete. Now this means nothing else than that the complete system of technical development and implementation has to be considered as well as the security/risk/profit psychology of the individual with its invironment and the autopoietic subsystem organization of society with its environment. On first sight this insight to take a holistic viewpoint and make that the basis for solving the issues involved with the renewable energy, grid IT, or any other complex exploitation network seems natural and in principle completely obvious - actually not even to be worth thinking about. However, the fundamental issues and challenges faced in exploitation networks to be implemented to reach Sustained Renewability, i.e. to solve the problems of resource network limitations and thus to overcome the fundamental limits of energetic and material consumption growth reaching carrying capacity limits by the classic approach, are obviously there and demand urgent solutions in respect due to the urge of the problems involved if nothing substantial is changed. Thus, the pure existence of the climate challenge shows the importance of a Sustained Renewability approach, which is characterized by investigating all detailed factors involved for a certain resource and the challenges of an exploitation super-network can be overcome. Thus, then the technical Tragedy of Systemic Complexity and the Inverse Tragedy of the Commons can be overcome since resources are not unsustainably overexploited but used in a Sustained Renewability way by holistically integrating the i) technical resource exploitation networks and ii) all autopoietic social subsystems on a macro level as well as the psychology of individuals on the micro level, in an holistically systemic ecology like manner - or in short in a Sustained Renewability approach.

\section{Conclusion}

The worldwide amount of energy and IT resources to sustain human life is - with the classic exploitation and usage strategies - fast reaching the limits and carrying capacity. Nevertheless, huge underused energy and IT resources are obviously available and often even renewable on a human scale. Therefore, the Tragedy of Systemic Complexity already on the technical level and the Inverse Tragedy of the Commons have to be overcome for the renewable energy as well as the grid IT sectors by combining solutions of the systemic resource and exploitation networks with solutions of all autopoietic social subsystems on a macro level as well as the psychology of individuals on the micro level, i.e. a technical systemic solution is combined with an extended Human Ecology paradigm. Thus, not only advanced underused resources can be implemented and internalized, but also Sustained Renewability, i.e. a long lasting resource exploitation and renewable cycles can be managed - for on human scales - large time spans with paradisiacal opportunities for the life on earth.

\section{Acknowledgements}

K. Egger and V. Baumgärtner are thanked for the discussions, which have lead to this work. My parents W. Knoch and W. F. Knoch are thanked for their contributions and support as well as: F. G. Grosveld, A. Abuseiris, N. Kepper, the German and 
International Societies for Human Ecology, the International Health Grid organization, the Erasmus Computing Grid, the German MediGRID and Services@MediGRID, the European EDGEs consortium, and the European EpiGenSys consortium. This work was supported by the Erasmus Medical Centre and the Hogeschool Rotterdam, The Netherlands, the BioQuant / German Cancer Research Centre (DKFZ), Germany, and the German Ministry for Education and Research (BMBF) under grant \# 01 AK 803 A-H (German MediGRID) and \# 01 IG 07015 G (German Services@MediGRID), the Dutch Ministry for Science and Education, the Netherlands Science Organization (NWO), the Britisch Biotechnology and Biological Sciences Research Council (BBSRC), the EraSysBio+ program (all EpiGenSys grant), and the European Commission (EpiGenSys and FP7 EDGEs grants) to TAK.

\section{References}

Bruckmeier, K. \& Serbser, W. H. (eds.) (2008). Ethik und Umweltpolitik: Humanökologische Positionen und Perspektiven. Oekom Verlag, ISBN 978-386-5811-19-6

de Zeeuw, L. V., Knoch, T. A., van den Berg, J. \& Grosveld, F. G. (2007). Erasmus Computing Grid - Het bouwen van een 20 TeraFLOP virtuelle supercomputer," In: H. Frederik (ed.), NIOC proceedings 2007 - het perspective of lange termijn, NIOC, Amsterdam, The Netherlands, pp. 52-59

Egger, K. \& Glaeser, B. (1975). Ideologiekritik der Grünen Revolution: Wege zur technologischen Alternative, Technologie und Politik. rororo actuell, Reinbeck

Egger, K. \& Rudolph, S. (1992). Zum anschaulichen Umgang mit komplexen Aspekten der Kultur- und Ökokrise. In: B. Glaeser and P. Teherani-Krönner, (eds.), Humanökologie und Kulturökologie - Grundlagen, pp. 191-220

Egger, K. (1996). Evolution, Menschenbild und Umweltkrise - ein Versuch zur humanökologischen Hypothesenbildung. In: H. Wehrt (ed.), Humanökologie Beiträge zum ganzheitlichen Verständnis unserer geschichtlichen Lebenswelt. Birkhäuser, Basel, pp. 155-189

Egger, K. (2008). Die moralische Inversion - Ursachen unserer normativen Orientierungskrise und deren Folgen für die Agrarentwicklung Alternativpositionen durch Dialog mit anderen Kulturen. In: K. Bruckmeier, and W. H. Serbser, (eds.), Ethik und Umweltpolitik: Humanökologische Positionen und Perspektiven. Oekom Verlag, ISBN 978-3-86581-119-6

Einstein, A. (1905). Ist die Trägheit eines Körpers von seinem Energieinhalt abhängig. Annalen der Physik, Vol. 323, No. 13, pp. 639-641

Faber, M., Stephan, G. \& Michaelis, P. (1989). Umdenken in der Abfallwirtschaft. Vermeiden, Verwerten, Beseitigen. Springer, ISBN 978-354-0518-39-6

Faber, M., Niemes, H., Stephan, G. \& Freytag, L. (1987). Entropy, Environment, and Resources. An Essay in Physico- Economics. Springer Verlag,ISBN 978-354-0182-48-1

Faber, M., Manstetten, R. \& Proops, J. (1998). Ecological Economics: Concepts and Methods. Edward Elgar Publishing, ISBN 978-185-8989-98-3

Faber, M. \& Manstetten, R. (2003) Mensch - Natur - Wissen: Grundlagen der Umweltbildung. Vandenhoeck and Ruprecht, ISBN 978-352-5301-41-8 
Faber, M. \& Schiller, J. (2006). Joint Production and Responsibility in Ecological Economics: On the Foundations of Environmental Policy (Advances in Ecological Economics). Edward Elgar Publishing, ISBN 978-184-0648-72-0

Foster, I. \& Kesselmann, C. (2004). The grid: blueprint for a new computing infrastructure. The Elsevier Series in Grid Computing. Morgan Kaufmann, Amsterdam and Boston, ISBN 1-5586-0933-4

Haeckel, E. (1866). Generelle Morphology der Organismen, Band 2, Allgemeine Entwicklungsgeschichte. Berlin, pp. 286

Haeckel, E. (1998). Natürliche Schöpfungsgeschichte. 9. Auflage, Berlin, pp. 793

Hardin, G. (1968). Tragedy of the Commons. Science, Vol. 162, pp. 1243-1248

Hardin, G. (1994). The Tragedy of the Unmanaged Commons. Trends in Ecology \& Evolution, Wol. 9, pp. 199

Hardin, G. (1998). Extensions of "The Tragedy of the Commons." Science, Vol. 280, pp. 682

International Association for the Study of the Commons (IASC), http:/ / www.iascp.org

IPCC: http://www.ipcc.ch and http://www.ipcc.ch/ ipccreports/index.htm

Jentzsch, W. I. (1995). BASF Antwerp - an integrated chemical plant. Industrial Lubrication and Tribology, Vol. 47, pp. 4-5

Knoch, T. A., Baumgärtner, V., de Zeeuw, L. V., Grosveld, F. G. \& Egger, K. (2009). e-Human Grid Ecology - understanding and approaching the inverse tragedy of the commons in the e-Grid Society. Studies in Health Technology and Informatics, Vol. 147, pp. 269-276

Knoch, T. A., Baumgärtner, V., Grosveld, F. G. \& Egger, K. (2010). Approaching the internalization challenge of grid technologies into e-Society by e-Human "Grid" Ecology. In J. Altmann, and O. F. Rana, (eds.), Economics of grids, clouds, and services, Springer, ISBN 978-364-2156-80-9, pp. 116-128

Krefting, D., Bart, J., Beronov, K., Dzhimova, O., Falkner, J., Hartung, M., Hoheisel, A., Knoch, T. A., Lingner, T., Mohammed, U., Peter, K., Rahm, E., Sax, U., Sommerfeld, D., Steinke, T., Tolxdorff, T., Vossberg, M., Viezens, F. \& Weisbecker, A. (2008). MediGRID - Towards a user friendly secured grid infrastructure. Future Generation Computer Systems, Vol. 25, pp. 326-336

Luhmann, N. (2004). Ökologische Kommunikation. VS Verlag für Sozialwissenschaften, ISBN 978-353-1517-75-9

Luhmann, N. (2008). Soziale Systeme: Grundriß einer allgemeinen Theorie. Suhrkamp, ISBN 978351-8282-66-3

Maturana, H. R. \& Varela, F. (1992). Tree of Knowledge. Shambhala, ISBN 978-087-7736$42-4$

Noether, E. (1918). Invariante Variationsprobleme. Gött. Nachr., pp. 235-257

Noether, E. (1918). Invarianten beliebiger Differentialausdrücke. Gött. Nachr., pp. 37-44

Ostrom, E. (1990). Governing the commons: The evolution of institutions for collective action. Cambridge University Press, ISBN 978-052-1405-99-7

Sax, U., Weisbecker, A., Falkner, J., Viezens, F., Yassene, M., Hartung, M., Bart, J., Krefting, D., Knoch, T. A. \& Semler, S. C. (2007). “Grid-basierte Services für die elektronische 
Patientenakte der Zukunft," E-HEALTH-COM - Magazin für Gesundheitstelematik und Telemedizin, Vol. 4, pp. 61-63

Sax, U., Weisbecker, A., Falkner, J., Viezens, F., Mohammed, Y., Hartung, M., Bart, J., Krefting, D., Knoch, T. A. \& Semler S. C. (2008). Auf dem Weg zur individualisierten Medizin - Grid-basierte Services für die EPA der Zukunft. In A. Jäckel (ed.), J Telemedizinführer Deutschland 2008. Deutsches Medizinforum, Minerva KG, Darmstadt, ISBN 978-393-7948-06-5, pp. 47-51

Tragedy of the commons, http://en.wikipedia.org/ wiki/Tragedy_of_the_commons 


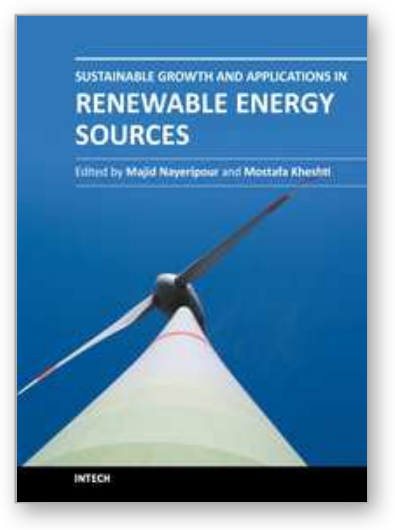

\section{Sustainable Growth and Applications in Renewable Energy Sources \\ Edited by Dr. Majid Nayeripour}

ISBN 978-953-307-408-5

Hard cover, 338 pages

Publisher InTech

Published online 02, December, 2011

Published in print edition December, 2011

Worldwide attention to environmental issues combined with the energy crisis force us to reduce greenhouse emissions and increase the usage of renewable energy sources as a solution to providing an efficient environment. This book addresses the current issues of sustainable growth and applications in renewable energy sources. The fifteen chapters of the book have been divided into two sections to organize the information accessible to readers. The book provides a variety of material, for instance on policies aiming at the promotion of sustainable development and implementation aspects of RES.

\section{How to reference}

In order to correctly reference this scholarly work, feel free to copy and paste the following:

Tobias A. Knoch (2011). Sustained Renewability: Approached by Systems Theory and Human Ecology, Sustainable Growth and Applications in Renewable Energy Sources, Dr. Majid Nayeripour (Ed.), ISBN: 978953-307-408-5, InTech, Available from: http://www.intechopen.com/books/sustainable-growth-andapplications-in-renewable-energy-sources/sustained-renewability-approached-by-systems-theory-and-humanecology

\section{INTECH}

open science | open minds

\section{InTech Europe}

University Campus STeP Ri Slavka Krautzeka 83/A 51000 Rijeka, Croatia Phone: +385 (51) 770447 Fax: +385 (51) 686166 www.intechopen.com

\section{InTech China}

Unit 405, Office Block, Hotel Equatorial Shanghai No.65, Yan An Road (West), Shanghai, 200040, China 中国上海市延安西路65号上海国际贵都大饭店办公楼405单元 Phone: +86-21-62489820

Fax: +86-21-62489821 
(C) 2011 The Author(s). Licensee IntechOpen. This is an open access article distributed under the terms of the Creative Commons Attribution 3.0 License, which permits unrestricted use, distribution, and reproduction in any medium, provided the original work is properly cited. 